\title{
Emerging Treatment Options for Acute Lymphoblastic Leukemia: Focus on CAR T-Cell Therapy
}

\author{
Presented by Patrick A. Brown, MD, and Bijal Shah, MD
}

\begin{abstract}
Acute lymphoblastic leukemia (ALL) comprises a heterogeneous group of diseases with different morphologic, cytogenetic, and molecular subgroups, some of which have significant therapeutic implications. It typically presents with an aggressive clinical course, and among adults, responds poorly to standard chemotherapy, and carries a high risk for relapse. Despite the significant progress made in inducing remission, frequent relapses remain a challenge. Novel drugs, such as potent later-generation tyrosine kinase inhibitors, antibody-drug conjugates, bispecific monoclonal antibodies, and chimeric antigen receptor (CAR) T-cell therapies, are being investigated in patients with ALL. This summary describes therapies currently approved for the treatment of patients with ALL, identifies emerging targeted immunotherapies for patients with ALL, and discusses adverse events and mechanisms of resistance.
\end{abstract}

J Natl Compr Canc Netw 2018;16(5.5):651-655 doi: 10.6004ljncen.2018.0048

Hope remains for patients with acute lymphoblastic leukemia (ALL)-hope to extend life and hope to minimize relapse-according to Patrick A. Brown, MD, and Bijal Shah, MD, who discussed the latest treatment options for ALL during their presentations at the NCCN 23rd Annual Conference.

"In just the past few years, progress with chimeric antigen receptor (CAR) T-cell therapies has greatly accelerated, with researchers developing a better understanding of how these therapies work in patients, and translating that knowledge into improvements in how they are developed and tested," noted Dr. Brown, Director, Pediatric Leukemia Program and Associate Professor of Oncology, The Sidney Kimmel Comprehensive Cancer Center at Johns Hopkins University, and Chair of the NCCN ALL Panel. "For

\footnotetext{
Presented by Patrick A. Brown, MD, The Sidney Kimmel Comprehensive Cancer Center at Johns Hopkins University, Baltimore, Maryland, and Bijal Shah, MD, Lymphoid Malignancies Section, Moffitt Cancer Center Tampa, Florida.

Dr. Brown has disclosed that he has received consulting fees/honoraria from Amgen Inc., Novartis Pharmaceuticals Corporation, and Shire. Dr. Shah has disclosed that he has received consulting fees/honoraria from Adaptive Biotechnologies and grant/research support from Incyte Corporation.

Correspondence: Patrick A. Brown, MD, The Sidney Kimmel Comprehensive Cancer Center at Johns Hopkins University, 1650 Orleans Street, CRB1 room 2M51, Baltimore, MD 21231.

Email: pbrown2@jhmi.edu; and

Bijal Shah, MD, Lymphoid Malignancies Section, Moffitt Cancer Center 12902 Magnolia Drive, FOB3-Heme, Tampa, FL 33647.

Email: bijal.shah@moffitt.org
}

years, the foundations of cancer treatment were surgery, chemotherapy, and radiation therapy. In 2017, 2 CAR T-cell therapies were FDA-approved: one for the treatment of children with ALL and the other for adults with advanced lymphomas."

For Dr. Shah, a hematologist/oncologist within the Lymphoid Malignancies Section, Moffitt Cancer Center and Vice Chair of the NCCN ALL Panel, hope should be balanced with a better understanding of the risks and benefits associated with these new immunotherapies. "CAR T-cell therapy has transformed the therapeutic options for patients with ALL, but there is still more work ahead that needs to be completed."

\section{Overview of ALL}

ALL comprises $80 \%$ of childhood leukemia and $15 \%$ to $20 \%$ of adult leukemia. It is estimated that in 2018 , there will be 6,590 new cases of ALL. ${ }^{1}$ Cure rates in ALL have improved dramatically over the past several decades, particularly among children. With current treatment regimens, the cure rate is approximately $80 \%$ for children and approximately $40 \%$ for adults. ${ }^{2}$ After relapse, the 5 -year overall survival rates are $20 \%$ to $30 \%$ in children, and even lower in adults.,4

"ALL is an uncommon leukemia in adults," Dr. Brown said. "Its treatment poses many challenges and requires expertise and experience in a number of medical 
Brown and Shah

disciplines and supportive care. We recommend that patients with ALL be referred to specialized treatment centers and, if possible, enrolled in clinical trials."

Dr. Brown also noted a contrast in the understanding of blood cancers compared with solid tumors. "We know so much more about leukemia on a genetic and molecular level than we do about solid tumors. That's because leukemia often invades the bloodstream rather than resulting in solid tumors. Thus, researchers need only to draw blood, rather than surgically remove part of a tumor, to study it."

For the clinicians in attendance, Dr. Brown summarized the therapies currently approved for the treatment of patients with Philadelphia chromosome (Ph)-positive and Ph-negative ALL. In addition, Dr. Brown identified emerging targeted immunotherapies and provided guidelines and strategies to incorporate them into the management of ALL.

\section{Standard Treatment Approaches for Relapsed and Refractory ALL}

Standard upfront treatment of adult ALL currently includes combination chemotherapy, with tyrosine kinase inhibitor (TKI) therapy added for Ph-positive ALL. This is often followed by stem cell transplant (SCT). According to Dr. Brown, there is an unmet need in patients with relapsed or refractory ALL, given that approximately $20 \%$ of pediatric patients and $60 \%$ of adult patients experience relapse after an initial complete response to conventional therapies, and median survival after relapse is typically short.

Until recently, the standard treatment for relapsed or refractory ALL was limited to similar approaches used upfront. With the development of immunotherapy, however, the options have expanded to include monoclonal antibody therapy (blinatumomab or inotuzumab ozogamicin) and CAR T-cell therapy (tisagenlecleucel) (Figure 1). ${ }^{1}$ If these therapies achieve a remission, the standard is to offer allogeneic SCT to patients with a suitable performance status and donor.

Inotuzumab ozogamicin is a humanized antiCD22 monoclonal antibody bound to a toxic natural calicheamicin. ${ }^{5}$ The first phase II study with inotuzumab ozogamicin conducted by Kantarjian et $\mathrm{al}^{6}$ provided patients with heavily pretreated ALL the opportunity to proceed to allogeneic SCT. Inotuzumab is generally well tolerated, although there is a significant incidence of veno-occlusive disease, especially in patients with prior or subsequent SCT. ${ }^{6}$

Blinatumomab is a bispecific CD19-directed CD3 T-cell engager that facilitates immune-mediated clearance of B-cell lymphoblastic leukemia (Figure 2). Approval is based on phase III randomized data demonstrating a 3-month median survival benefit. Additionally, recently published data have allowed for broadening of the label to include patients with minimal residual disease (MRD), making blinatumomab the first and only agent approved for those with $\geq 0.1 \%$ leukemic blasts. Notably, immune activation was accompanied by both cytokine release syndrome and neurologic toxicities - a forerunner to events that would characterize CAR T-cell therapy. Mechanisms of resistance to blinatumomab have been difficult to discern, although post hoc subgroup analysis suggests that both the number of prior therapies and tumor bulk appear to play a role.

"It was surprising to me," said Dr. Shah, "that the number of prior therapies before blinatumomab, particularly in the context of MRD, would influence the outcome significantly, and I wonder if this observation may suggest an optimal window to advance immunotherapy prior to T-cell exhaustion and senescence."

Dr. Brown noted that superior outcomes have been achieved with pediatric protocols in young adults. Consequently, the NCCN Guidelines stress "pediatric-inspired" treatment regimens should be considered for adolescent and young adult (AYA) patients. The AYA age group is generally considered to be 18 to 39 years, but the NCCN Guidelines note that "chronologic age is a poor surrogate for fitness for therapy, and patients should be evaluated on an individual basis."

\section{CAR T-Cell Therapies}

"CAR T-cell therapy is unique among treatments because it simultaneously serves as a cell therapy, gene therapy, and immunotherapy," noted Dr. Shah. As part of his presentation, Dr. Shah centered on the newer therapies for patients with ALL: tisagenlecleucel and axicabtagene ciloleucel. Both therapies require leukapheresis, in which patients' $T$ cells are removed from their peripheral blood. After removal, the cells must undergo genetic modification, typically using a retrovirus or 


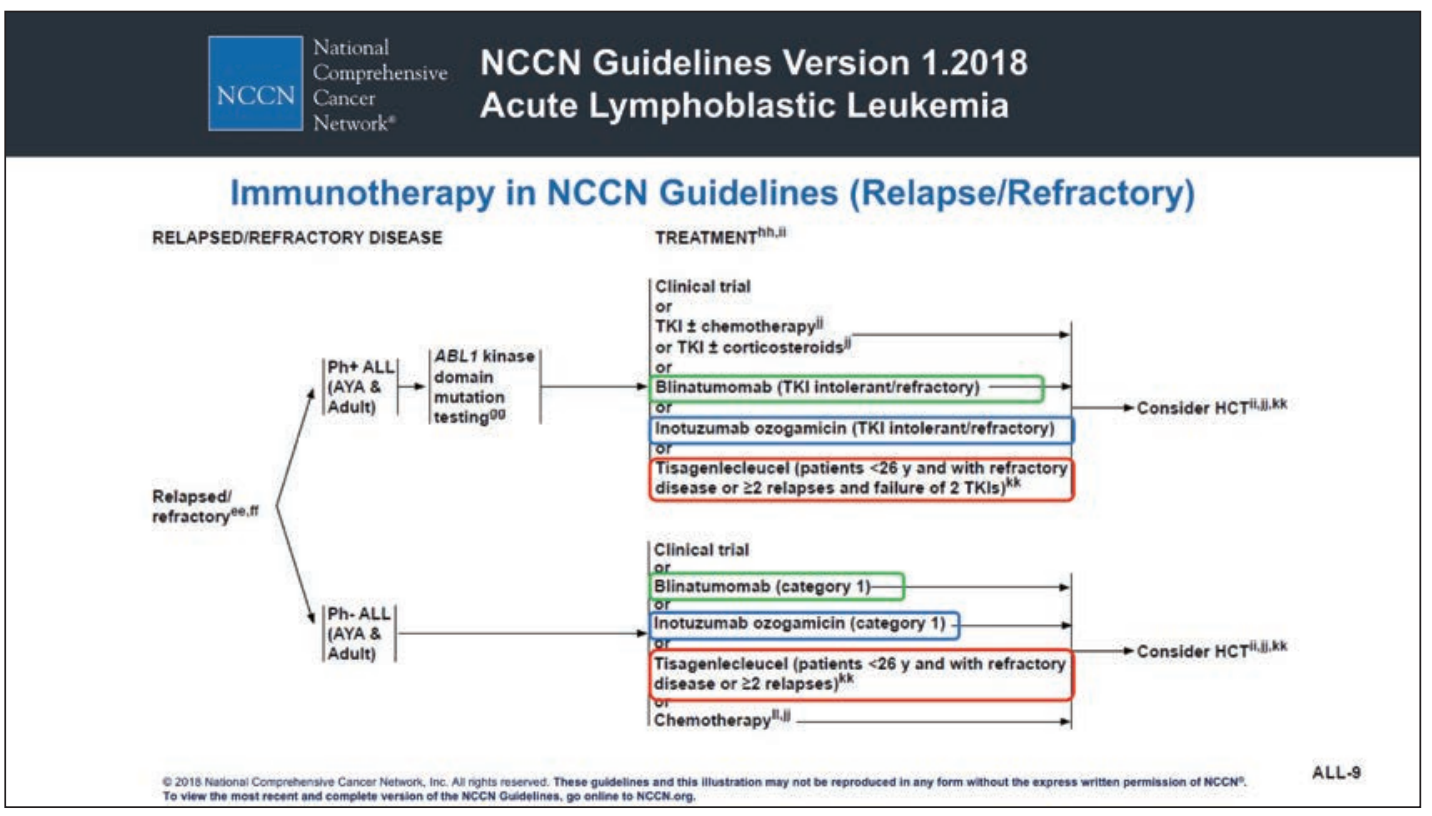

Figure 1. Immunotherapy for relapsed or refractory acute lymphoblastic leukemia.

lentivirus, to produce a "chimeric antigen receptor," or CAR. The CAR possesses 3 major functional domains: an immunoglobin-like protein that binds CD19 (on leukemic blasts), coupled to CD3-zeta, and a costimulatory protein (either CD28 or 4-1BB) to facilitate activation of the $\mathrm{T}$ cell. After it is produced, the patient must undergo conditioning chemotherapy to both reduce tumor burden and deplete residual $\mathrm{T}$ cells, to allow the CAR T cells to better grow. After the conditioning chemotherapy, the patient undergoes infusion of CAR T cells.

Tisagenlecleucel is a CD19-directed, genetically modified, autologous T-cell immunotherapy using a 4-1BB costimulatory domain. Tisagenlecleucel is indicated for the treatment of patients aged $<26$

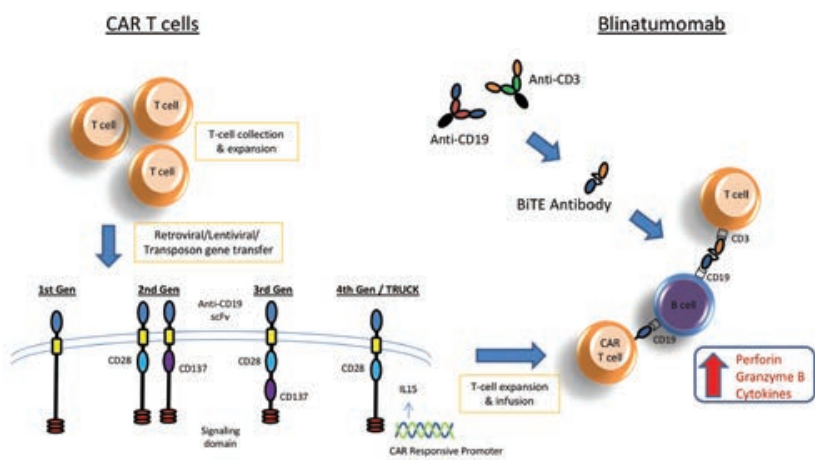

Figure 2. T-cell-directed therapy in B cell acute lymphoblastic leukemia. years with B-cell precursor ALL that is refractory or in second or later relapse. ${ }^{7}$ Three single-arm trials of tisagenlecleucel for pediatric ALL are noteworthy. ${ }^{8,9}$ The first, B2101J, was a single-site phase I/IIa trial that used split dosing of CAR T cells manufactured at the University of Pennsylvania. The subsequent multicenter trials (a single-site University of Pennsylvania study and the pivotal multicenter JULIET trial) used a single infusion of CAR T cells at the current standard dose. ${ }^{8,9}$

Axicabtagene is a CD19-directed, genetically modified, autologous T-cell immunotherapy using a CD28 costimulatory domain. It is currently under investigation for adults with relapsed or refractory ALL. Preliminary data from the phase I study support a high MRD-negative response rate (88\%), and further phase II testing is expected to commence in $2018 .{ }^{10}$

\section{Relapse and Role of Tumor Burden}

Both Dr. Brown and Dr. Shah focused on how tumor burden played a significant role in influencing patient outcomes. "When we think about those variables that influence outcome, there's no question tumor burden plays a significant role," said Dr. Shah. "Again, this was another surprise for me. My own perception was that immunotherapy would be 
Brown and Shah

able to overcome this risk factor. But in the case of blinatumomab, we still saw significant risk associated with high tumor burden in the marrow."

Dr. Shah discussed the impact of tumor burden on adverse events. "What we see as it relates to toxicity is without question that marrow burden drives the increase in CAR T-cell peak, and that in turn is associated with an increase in adverse events. That's not to say that the CAR T-cell dose doesn't also play a role in terms of toxicity, but as far as we can tell, the CAR T-cell dose has not been associated with an increase in responses. That is, going up on the dose doesn't necessarily improve the complete response rate," he said.

For clinicians, the challenge moving forward is to determine what is driving the relapses, particularly in adult patients. For Dr. Shah, the answer may lead to knowing "what should be the best choice of therapies." Unfortunately, a significant subset of patients still experience relapse, and many of these relapses (after blinatumomab or CART-19 therapy) are characterized by the apparent loss of CD19.1" "The mechanism of this escape is still under investigation," stated Dr. Shah, "although some reports suggest that convergence of acquired mutations and alternative splicing of CD19 enables leukemic cells to resist CART-19 immunotherapy."

\section{Toxicity}

Before discussing CAR T-cell toxicity, it is important to stress the process, which necessarily includes needed time to collect and manufacture the CAR $\mathrm{T}$ cells prior to infusion. "If you have a 3- to 4-week manufacturing time for the CAR T cells, or even a 2-week manufacturing time, you recognize that some patients are just going to get very sick before you're actually able to perfuse cells. So it's going to be absolutely critical that we address this," Dr. Shah said.

CAR T-cell-associated toxicities, such as cytokine-release syndrome (CRS) and neurologic events, now well-recognized complications of immunotherapy, are important to recognize and appropriately treat. "I don't think we should talk about CAR T-cell therapy without acknowledging the toxicity," said Dr. Shah. "I think we have to be cognizant of the fact that these therapies will make people sick in the short term."
As for CRS, more common symptoms include fever and hypotension. However, some patients may experience more severe symptoms that could lead to potentially life-threatening complications, such as cardiac dysfunction, acute respiratory distress syndrome, and/or multiorgan failure. ${ }^{12}$ "As we get into more severe CRS, what we're really talking about is hypotension requiring vasopressors, hypoxia requiring oxygen supplementation, and then ultimately, the need for intubation and [occurrence of] more significant organ compromise." Both Dr. Brown and Dr. Shah commented that these adverse events remain manageable for most patients. "In many patients, both children and adults, CRS can be managed with standard supportive therapies, including tocilizumab and steroids," stated Dr. Brown.

Tocilizumab is a monoclonal antibody targeting the interleukin 6 receptor (IL6-R), a key mediator of CRS, and is approved as an intravenous injection for the treatment of CAR T-cell-induced severe or life-threatening CRS in patients aged $\geq 2$ years. ${ }^{13}$ Although tocilizumab is readily used in clinical practice to control some of the associated side effects, it has its limitations. "It's helpful in managing CRS," stated Dr. Shah, "but it's not helpful with neurotoxicity, which may respond better to steroids." Similarly, for severe cases that do not respond quickly to tocilizumab, steroid administration to suppress the CAR T-cell expansion can prove critical. With supportive care, the overall risk of dying from CAR T-cell therapy is low (approximately 3\%-5\%), and most patients will recover to baseline within a few weeks. ${ }^{14,15}$

Unfortunately, despite high response rates with both tisagenlecleucel and axicabtagene, management and prevention of relapse remains a concern. According to Dr. Shah, "A really surprising thing to me was that although we think about allotransplant as the approach we should take for patients who [experience] remission, as it turns out, on clinical trials, only about one-third of patients are making that transition." Further adding to this confusion is a failure to demonstrate any improvement in survival when censoring for transplanted individuals. "Although these analyses do not meet the standard of a 'donor versus no donor' trial, they do force one to question the magnitude of benefit, particularly given the inherent risks of SCT," he said. 
Acute Lymphoblastic Leukemia

\section{References}

1. Brown PA, Shah B, Advani A, et al. NCCN Clinical Practice Guidelines in Oncology (NCCN Guidelines): Acute Lymphoblastic Leukemia. Version 1.2018. Accessed April 7, 2018. To view the most recent version of the guidelines, visit NCCN.org.

2. Cancer Research UK. Acute lymphoblastic leukemia. Available at: http:// www.cancerresearchuk.org/about-cancer/acute-lymphoblastic-leukaemiaall/survival. Accessed April 7, 2018.

3. Crotta A, Zhang J, Keir C. Survival after stem-cell transplant in pediatric and young-adult patients with relapsed and refractory B-cell acute lymphoblastic leukemia. Curr Med Res Opin 2018;34:435-440.

4. Locatelli F, Testi AM, Bernardo ME, et al. Clofarabine, cyclophosphamide and etoposide as single-course re-induction therapy for children with refractory/multiple relapsed acute lymphoblastic leukaemia. Br J Haematol 2009;147:371-378.

5. George B, Kantarjian H, Jabbour E, et al. Role of inotuzumab ozogamicin in the treatment of relapsed/refractory acute lymphoblastic leukemia. Immunotherapy 2016;8:135-143.

6. Kantarjian H, Thomas D, Wayne AS, et al. Monoclonal antibody-based therapies: a new dawn in the treatment of acute lymphoblastic leukemia. J Clin Oncol 2012;30:3876-3883.

7. Novartis. Tisagenlecleucel PI. Available at: https://www.pharma. us.novartis.com/sites/www.pharma.u s.novartis.com/files/kymriah.pdf. Accessed April 7, 2018.

8. Novartis. Tisagenlecleucel (CTLO19) for the treatment of pediatric and young adult patients with relapsed/refractory B-cell acute lymphoblastic leukemia. FDA Advisory Committee Briefing Document.
Available at: https://www.fda.gov/downloads/advisorycommittees/ committeesmeetingmaterials/drugs/oncologicdrugsadvisorycommittee/ ucm566168.pdf. Accessed April 7, 2018.

9. Schuster SJ, Bishop MR, Tam CS, et al. Primary analysis of Juliet: a global, pivotal, phase 2 trial of CTL019 in adult patients with relapsed or refractory diffuse large B-cell lymphoma. Blood 2017;130 (Suppl 1):577.

10. Neelapu SS, Locke FL, Bartlett NL, et al. Axicabtagene ciloleucel CAR T-cell therapy in refractory large B-cell lymphoma. N Engl J Med 2017;377:2531-2544.

11. Ruella M, Mau MV. Catch me if you can: leukemia escape after CD19. directed T-cell immunotherapies. Comp Struct Biol J 2016;14:357-362.

12. Lee DW, Gardner R, Porter DL, et al. Current concepts in the diagnosis and management of cytokine release syndrome. Blood 2014;124:188-195.

13. Genentech News Release. FDA Approves Genentech's Actemra (Tocilizumab) for the Treatment of CAR T Cell-Induced Cytokine Release Syndrome. Available at https://www.gene.com/media/pressreleases/14679/2017-08-30/fda-approves-genentechs-actemra-tocilizumab. Accessed April 7, 2018.

14. Neelapu SS, Locke FL, Bartlett NL, et al. Axicabtagene ciloleucel CAR T-cell therapy in refractory large B-cell lymphoma. N Engl J Med 2017;377:2531-2544.

15. Maude SL, Laetsch TW, Buechner J, et al. Tisagenlecleucel in children and young adults with B-cell lymphoblastic leukemia. N Engl J Med 2018;378:439-448. 